\title{
Acute flaccid myelitis and enterovirus D68: lessons from the past and present
}

\author{
Jelte Helfferich ${ }^{1}$ (D) Marjolein Knoester ${ }^{2}$ - Coretta C. Van Leer-Buter ${ }^{2} \cdot$ Rinze F. Neuteboom ${ }^{3} \cdot$ Linda C. Meiners $^{4}$. \\ Hubert G. Niesters ${ }^{2}$ - Oebele F. Brouwer ${ }^{1}$
}

Received: 19 April 2019 / Revised: 11 July 2019 / Accepted: 16 July 2019 / Published online: 23 July 2019

(C) The Author(s) 2019

\begin{abstract}
Acute flaccid myelitis is characterized by the combination of acute flaccid paralysis and a spinal cord lesion largely restricted to the gray matter on magnetic resonance imaging. The term acute flaccid myelitis was introduced in 2014 after the upsurge of pediatric cases in the USA with enterovirus D68 infection. Since then, an increasing number of cases have been reported worldwide. Whereas the terminology is new, the clinical syndrome has been recognized in the past in association with several other neurotropic viruses such as poliovirus.

Conclusion: This review presents the current knowledge on acute flaccid myelitis with respect to the clinical presentation and its differential diagnosis with Guillain-Barré syndrome and acute transverse myelitis. We also discuss the association with enterovirus D68 and the presumed pathophysiological mechanism of this infection causing anterior horn cell damage. Sharing clinical knowledge and insights from basic research is needed to make progress in diagnosis, treatment, and prevention of this new polio-like disease.

What is Known:

- Acute flaccid myelitis (AFM) is a polio-like condition characterized by rapid progressive asymmetric weakness, together with specific findings on MRI

- AFM has been related to different viral agents, but recent outbreaks are predominantly associated with enterovirus D68.

What is New:

- Improving knowledge on AFM must increase early recognition and adequate diagnostic procedures by clinicians.

- The increasing incidence of AFM urges cooperation between pediatricians, neurologists, and microbiologists for the development of treatment and preventive options.
\end{abstract}

Keywords Acute flaccid myelitis · Acute flaccid paralysis $\cdot$ Enterovirus D68 $\cdot$ Poliovirus $\cdot$ Poliomyelitis $\cdot$ Enterovirus

Communicated by Mario Bianchetti

Electronic supplementary material The online version of this article (https://doi.org/10.1007/s00431-019-03435-3) contains supplementary material, which is available to authorized users.

Jelte Helfferich

j.helfferich@umcg.nl

Marjolein Knoester

m.knoester@umcg.nl

Coretta C. Van Leer-Buter

c.van.leer@umcg.nl

Rinze F. Neuteboom

r.neuteboom@erasmusmc.nl

Linda C. Meiners

1.c.meiners@umcg.nl

Hubert G. Niesters

h.g.m.niesters@umcg.nl
Oebele F. Brouwer

o.f.brouwer@umcg.nl

1 Department of Neurology, University Medical Center Groningen, University of Groningen, Hanzeplein 1, PO Box 30001, 9700 RB Groningen, The Netherlands

2 Department of Medical Microbiology and Infection Prevention, Division of Clinical Virology, University Medical Center Groningen, University of Groningen, Groningen, The Netherlands

3 Department of Pediatric Neurology, Erasmus Medical Center, Erasmus University, Rotterdam, The Netherlands

4 Department of Radiology, University Medical Center Groningen, University of Groningen, Groningen, The Netherlands 


$\begin{array}{ll}\text { Abbreviations } \\ \text { ADEM } & \text { Acute disseminated encephalomyelitis } \\ \text { AFM } & \text { Acute flaccid myelitis } \\ \text { AFP } & \text { Acute flaccid paralysis } \\ \text { ATM } & \text { Acute transverse myelitis } \\ \text { CDC } & \text { Center for Disease Control and Prevention } \\ \text { CMAP } & \text { Compound muscle action potential } \\ \text { CSF } & \text { Cerebrospinal fluid } \\ \text { EMG } & \text { Electromyography } \\ \text { EV-A71 } & \text { Enterovirus A71 } \\ \text { EV-D68 } & \text { Enterovirus D68 } \\ \text { GBS } & \text { Guillain-Barré syndrome } \\ \text { IVIG } & \text { Intravenous immunoglobulin } \\ \text { MRI } & \text { Magnetic resonance imaging } \\ \text { WNV } & \text { West Nile virus }\end{array}$

\section{Introduction}

Acute flaccid myelitis (AFM) is a syndrome characterized by acute flaccid paralysis (AFP) and gray matter spinal cord lesions on magnetic resonance imaging (MRI). After the introduction of the term AFM in 2014, more than 500 patients, predominantly children, have been recognized both in- and outside Europe [5, 27, 32, 48].

The Center for Disease Control and Prevention (CDC) proposed a case definition in which a definite AFM case is described as acute-onset flaccid weakness, combined with a spinal cord lesion on MRI, largely restricted to the gray matter and spanning one or more spinal segments. Acute flaccid weakness combined with cerebrospinal fluid (CSF) pleocytosis without lesions on MRI is defined as a probable case [4].

A prodromal illness, asymmetric limb weakness, and specific findings in electromyography and nerve conduction studies may further aid in distinguishing AFM from other causes of AFP such as Guillain-Barré syndrome (GBS) and acute transverse myelitis (ATM) [10].

Accumulating evidence supports an association between enterovirus D68 (EV-D68) and AFM [9, 34]. Other viruses that have been associated with outbreaks of acute flaccid weakness and myelitis include enterovirus A71 (EV-A71), West Nile virus (WNV), Japanese encephalitis virus, and the wild-type poliovirus [12, 22, 43, 45].

In this review, we describe the clinical syndrome of AFM, its differential diagnosis, and its association with different viruses, with the emphasis on EV-D68.

\section{Methods}

For this review, we performed a literature search in PubMed on "flaccid myelitis" and "Enterovirus D68" from 2000 until
February 2019. A total of 995 titles of articles in English were screened and selected based on relevance for epidemiology, clinical characteristics, pathophysiology, treatment, prevention, and prognosis of AFM. Only cohorts containing at least five children were selected (Table 1).

\section{Epidemiology}

Before the term AFM was introduced, outbreaks of acute flaccid weakness and myelitis, matching the case definition for AFM, were reported in association with EV-A71, predominantly in Eastern Asia and Australia, and with WNV, causing several outbreaks in the USA in the beginning of this century $[12,22]$. Poliomyelitis also matches the case definition of AFM and can be seen as the first known cause of AFM. However, MRI was and is often not available in countries where poliomyelitis still occurs, making the definite diagnosis of AFM difficult.

In 2012, the first probable cases were reported in California (USA) [10]. Since 2014, the CDC has reported over 500 cases of AFM in the USA with 2-year intervals and several cohorts of patients with AFM have been reported worldwide (Table 1) [5, 10, 11, 23, 24, 31, 39, 40, 42, 48]. A recent study reported an incidence of 1.46 per 100,000 person years, although reliable data is lacking, as AFM is notifiable in only few countries and the clinical picture is often not recognized [25].

In different cohorts of AFM patients, EV-D68 was detected in $20-40 \%$ of cases, primarily from respiratory specimens (Table 1). The variation in detection percentages might be explained by differences in timing and performance of diagnostic procedures and by selection criteria for patients [25]. Most reported patients with AFM were children under the age of 10 with a slight male preponderance. A majority were previously healthy, but asthma was seen in $12-32 \%$ of children $[25,33,48]$.

Both EV-A71 and West Nile virus are still circulating and have also been detected in recent cohorts of AFM patients [2, $3,31]$. Outbreaks of poliomyelitis are currently rare, due to a global poliovirus surveillance and vaccination program [8].

\section{Clinical features}

The clinical characteristics of non-polio AFM cohorts described in literature since 2012 are summarized in Table 1. Muscle weakness typically develops over the course of several hours to days, often with a marked asymmetry. Weakness is proximally usually more severe and may be more pronounced in the upper limbs, with a spectrum of severity varying between slight paresis of a single limb to tetraplegia. Tendon reflexes are typically diminished or absent in the affected limbs. In most patients, there is a prior prodromal illness, often involving the upper respiratory tract, with a median of 5 days before onset of weakness [5, 10, 27, 33]. 


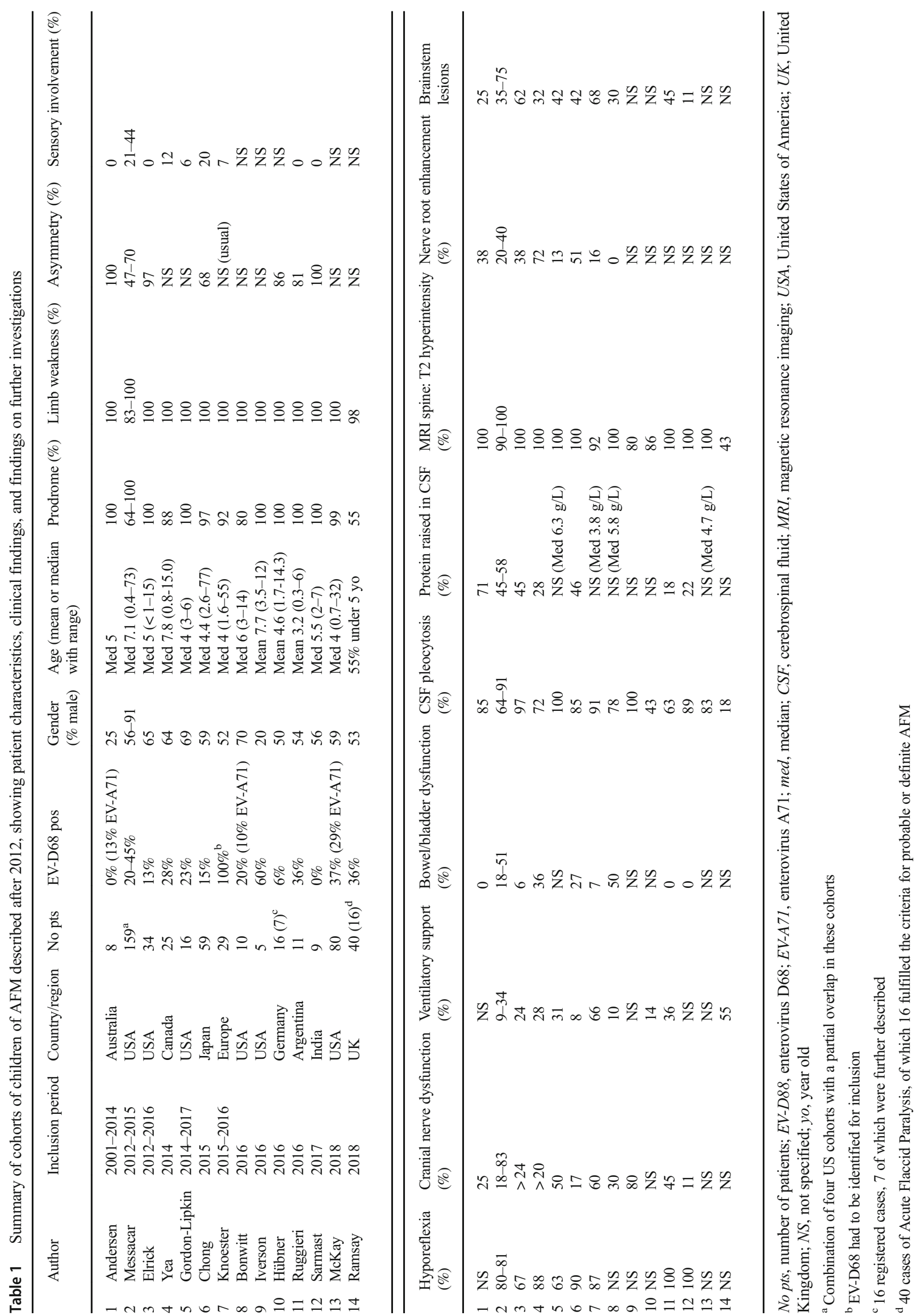


Weakness can be limited to the extremities, but the diaphragm and bulbar muscles may also be affected, making ventilatory support necessary in the acute phase in about $30 \%$ of cases $[10,13,33,48]$. Cranial nerve deficits are common and may be the only finding. The facial nerve is most often affected, followed by the abducens and oculomotor nerves [33].

Associated features include severe limb pain and autonomic disturbances such as bladder dysfunction. Sensory symptoms, primarily paresthesia, are reported in up to $20 \%$ of cases $[5,10,27,33]$.

The clinical features of cohorts of AFM, described before 2012, associated with EV-A71, WNV, and poliovirus were highly similar, although poliovirus-related AFM more often affected lower limbs, with bulbar muscles usually being spared [43]. EV-A71 has also been associated with rhombencephalitis, sometimes with severe cardiorespiratory symptoms $[12,22,43]$.

\section{Differential diagnosis}

AFM is included in the broad differential diagnosis of AFP. AFP is defined as a syndrome of focal weakness of peripheral origin in any part of the body with an acute onset [30].

It is important to be able to recognize AFM early in its course so that adequate diagnostic procedures can be performed and respiratory failure in the initial phase can be anticipated. Both clinical clues and findings on further investigations may help differentiate AFM from other causes of AFP.

In cases of AFM in which only one arm is affected, the initial thought may be that of synovitis or arm injury. Clinical clues that may help in distinguishing these from AFM may be the presence of a prodromal illness, the hypo- or areflexia, and the often-associated neck weakness in AFM.

When more than one limb is affected, the differential diagnosis includes other causes of acute myelopathy, such as acute transverse myelitis (ATM), acute disseminated encephalomyelitis (ADEM), acute cord compression, and ischemic myelopathy. Furthermore, Guillain-Barré syndrome (GBS) may be suspected because of the sudden onset of flaccid weakness after a prodromal illness.

While the asymmetric weakness, the absence of encephalopathy, the paucity of sensory symptoms, and the presence of cranial nerve deficits in AFM may help in distinguishing it from other causes of AFP, further investigations are required to make the right diagnosis (Table 2; Figs. 1, 2, 3, 4) [1, 10, 20, $33,47]$.

\section{Investigations}

Diagnostic tests recommended in children with suspected AFM should be directed at the identification of different microorganisms and the exclusion of other causes (Table 3) [15].
Initial investigations must be performed on blood, stool, respiratory material, and CSF, followed by MRI of the brain and spinal cord and in some cases electromyography (EMG).

\section{Blood}

General laboratory investigation of blood samples of AFM patients may show a slight leukocytosis, sometimes with raised inflammatory parameters, which is usually not helpful in the differentiation of AFM from other disorders causing AFP [20, 33].

\section{Cerebrospinal fluid}

CSF examination in AFM patients in the described cohorts since 2012 reveals a mild to moderate pleocytosis in most cases (Table 1). Protein levels are initially minimally raised in about half of AFM cases but can be completely normal. After several days, the leukocyte number tends to decrease, while protein levels rise $[5,14,27,33]$. Oligoclonal bands in the CSF can be identified in immune-mediated conditions such as ATM, but are usually not found in AFM [1].

Interestingly, viral agents, such as EV-D68, EV-A71, and poliovirus, are only detected in the CSF in a small minority of patients with AFM [5, 22, 27, 40, 43].

\section{Virology diagnostic testing}

The viral RNA of EV-D68 is detected mostly in respiratory samples, followed to a much lesser extent by feces and can only rarely be found in blood or CSF. This in contrast to EVA71, which is more frequently detected in blood, and poliovirus, which is routinely identified in stool samples [15, 43].

Obtaining an adequate respiratory sample is therefore indispensable for detection of EV-D68. Considering the fact that the prodromal, mostly respiratory illness is usually a few days into its natural course when a patient presents with weakness, the best chances of detecting EV-D68 is soon after onset of complaints. Several PCR tests have been described, which test either directly for EV-D68 or for enteroviruses in general [15, 38].

\section{Magnetic resonance imaging}

MRI of the brain and spinal cord is important in making the diagnosis of AFM and in distinguishing it from other causes of AFP (Table 2 and Figs. 1, 2, 3, 4) [13]. CT usually shows no abnormalities [28].

In AFM, the classical MRI feature is a longitudinally extensive slight T2-hyperintense signal in the central cord, affecting the central gray matter, often most pronounced in the cervical regions (Fig. 1b, c). Initially, there is usually more diffuse spinal cord edema, evolving over several days to T2- 
hyperintensity that is restricted to the anterior horn. Enhancement of the caudal roots and sometimes of the cranial nerves can be seen (Fig. 1d) [28].

MRI of the brain commonly reveals an area of slight hyperintensity typically located in the dorsal pons in the region of the nuclei of the abducens and facial nerve. The corticospinal tracts, located ventrally, are not affected (Fig. 1a), while the caudate nucleus may be involved [28]. These findings may help in securing the diagnosis, but the correlation between symptoms and radiologic findings is usually poor, making MRI unsuitable as a prognostic tool for AFM [37].

Imaging findings in earlier outbreaks of AFM, associated with WNV and poliovirus, were highly similar, while in EVA71-associated neurological disease, these appear to be more variable and more extensive brain abnormalities may occur $[6$, $12,22]$.

\section{Neurophysiological studies}

While EMG findings in recent outbreaks of AFM can be normal on the first day, after several days, a pattern compatible with anterior horn disease is seen. This encompasses decreased compound muscle action potentials (CMAP) with normal conduction velocities. Sensory testing is usually completely normal [21].

After some weeks, denervation potentials can be seen, with severe ongoing denervation being a possible predictor for the gravity of residual damage. The persistence of F-waves may indicate a better prognosis $[5,21,33]$.

\section{Virology}

Enteroviruses, such as EV-D68, EV-A71, and poliovirus, are small RNA viruses belonging to the picornavirus family. EVD68 was first identified in 1962 after isolation from children with severe respiratory disease [44]. Since 2012, an increasing incidence has been recognized, with infections mostly occurring in autumn and late summer. EV-D68 appears to occur in a cyclic pattern with a 2-year interval $[27,34]$.

EV-D68 infection may be asymptomatic or cause respiratory disease. In hospitalized children, an asthma-like respiratory disease is most commonly seen [39]. The percentage of infected patients afflicted with paralytic disease is not yet known, but is estimated to be less than $1 \%$, similar to poliomyelitis [29, 43].

\section{Pathophysiology}

A causal relationship between EV-D68 and AFM is supported by epidemiological and biological evidence, as was evaluated by different groups applying the Bradford Hill criteria [9, 34].
The biological evidence mainly came from mouse models, in which mice infected with contemporary circulating strains of EV-D68 develop flaccid paralysis mimicking AFM. Interestingly, neonatal or young mice are used, because older mice are not susceptible to disease [17, 36]. Pathologic examination of infected mice revealed the presence of the virus in the anterior horn with associated cell loss [17, 36]. EV-D68 probably reaches the anterior horn by retrograde axonal transport, as is supported by both mouse studies and in vitro studies in human motor neurons $[17,19,36]$.

One study found myositis without spinal cord infection after intranasal injection of the virus in mice [36].

Although the results from mouse studies cannot simply be extrapolated to humans, these results are suggestive of a damaging effect of the virus in anterior horn cells, possibly combined with a direct damaging effect on muscles through viral myositis.

Important questions remain why only some EV-D68 infected patients develop AFM and how the variability in severity of AFM in affected patients is explained.

\section{Treatment}

There are currently no effective treatment options for AFM. Most patients are treated with intravenous immunoglobulin (IVIG), steroids, or plasmapheresis, or a combination, but no significant clinical effect of any of these interventions has been shown so far. Because of its effectiveness in the mouse model of EV-D68-associated AFM and its possible efficacy in treatment of EV-A71-associated encephalomyelitis, treatment with IVIG has been recommended [18, 33, 46, 48].

The anti-inflammatory effects of steroids may be beneficial in AFM cases with spinal cord edema or white matter involvement, but steroids are unlikely to be effective in limiting the anterior horn damage that is probably caused by a direct damaging effect of the virus. Furthermore, treatment with steroids in a mouse model of AFM associated with EV-D68 led to an increased viral load and a deterioration of motor symptoms $[17,36]$.

Fluoxetine, an antidepressant, is effective in inhibiting EVD68 replication in vitro. However, treatment with fluoxetine in the mouse model of EV-D68-associated AFM did not result in reduction of the viral load or improvement of motor function. Also, no significant effect has been shown in patients with AFM, treated with fluoxetine $[18,35]$.

While scientific proof is still lacking, we recommend IVIG in the acute phase, combined with maximal supportive care with optimal pain control, feeding, ventilatory support, and intensive rehabilitation. Surgical procedures such as nerve and muscle transfers have been performed and cases have been described in which improvement of limb function has been achieved. Because over time degeneration of the receiving motor nerves and muscle fibers will occur, evaluation for 


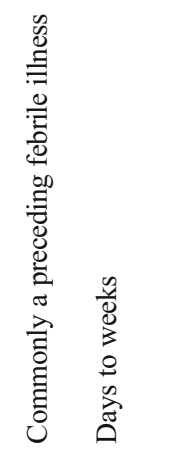<smiles>CC1[C@@H](C)[C@@H](C)C[C@H](C)[C@@H]1C</smiles>

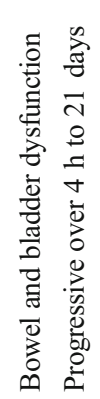

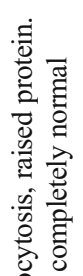

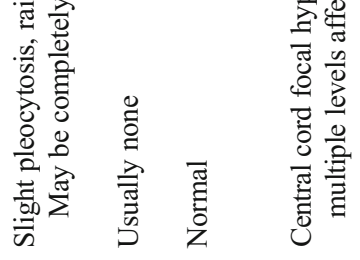

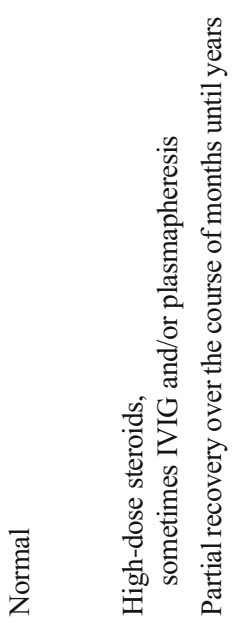

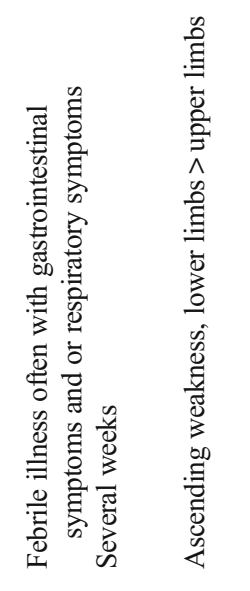

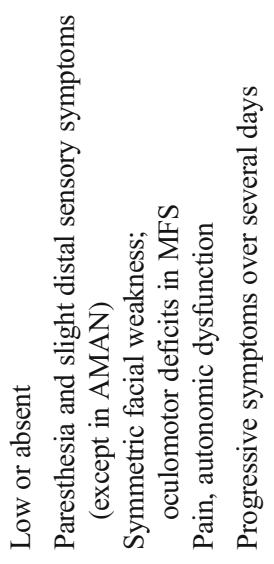

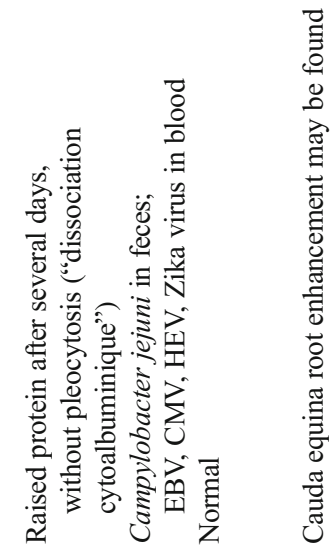

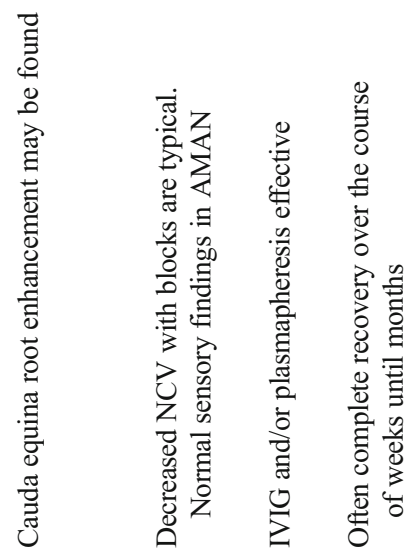

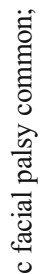<smiles>C1CCCC1</smiles>
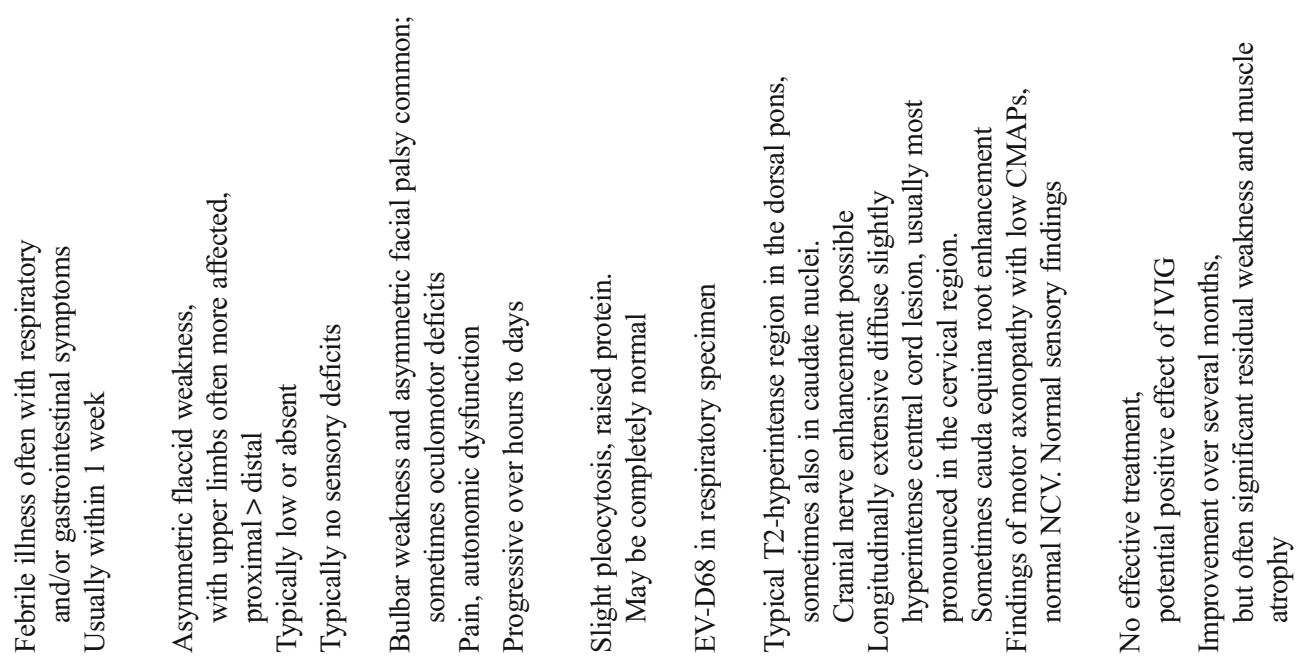

है

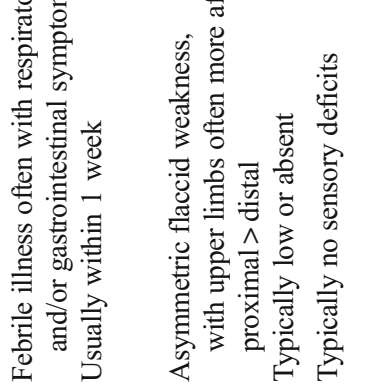

总

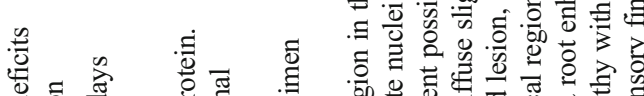

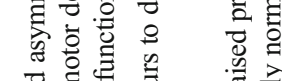

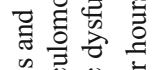

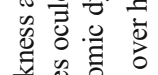

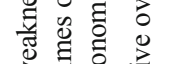

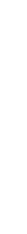
咅

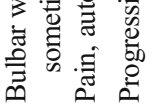

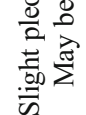

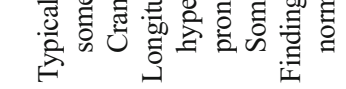

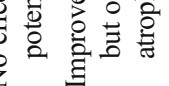

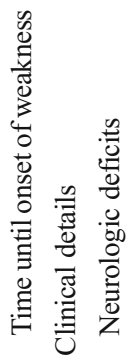

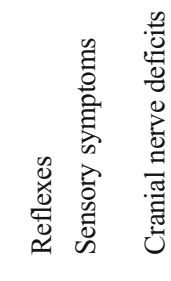

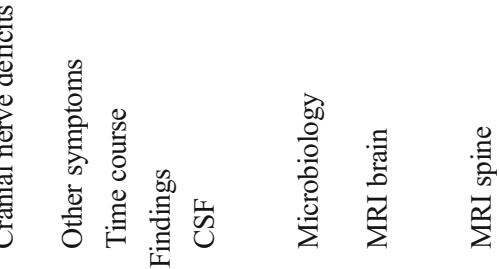<smiles>CC1[C@@H](C)C[C@@H]1C</smiles> 


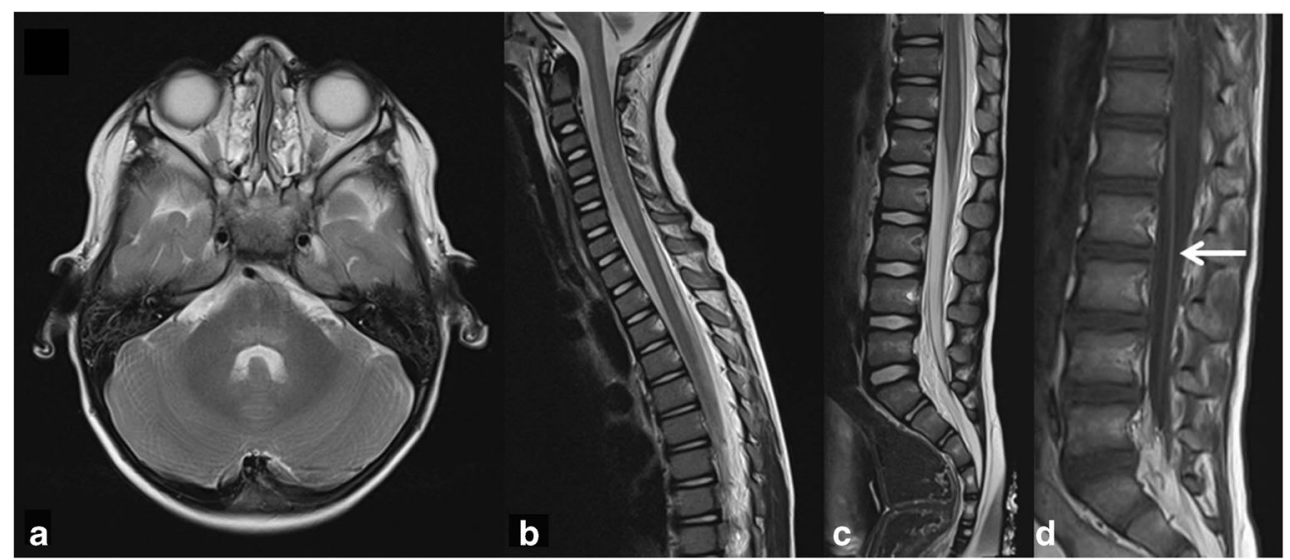

Fig. 1 MRI of the neuraxis in a 3-year-old boy with EV-D68-associated AFM. a Brain: transverse T2-weighted image showing an area of slight hyperintensity in the dorsal pons (arrow). b and $\mathbf{c}$ Spinal cord: sagittal T2weighted images showing longitudinal slight hyperintensity largely

surgical intervention should be considered early in the disease course [41].

\section{Prevention/vaccination}

In the mouse model of EV-D68-associated AFM, passive immunization with pooled immune sera, if administered before injection of the virus, was effective in decreasing the rate of paralysis [18].

Arguments for vaccination as a treatment strategy arise from the development of effective vaccines against EV-A71 restricted to the central cord, where the gray matter is situated (arrow). d Spinal cord: contrast enhancement of the ventral caudal roots on a sagittal T1-weigthed image (arrow) (republished with permission from [16])

infections in China and the effective eradication of poliomyelitis in most of the world after introduction of vaccination [8, 49]. Recently, an experimental vaccine based on virus-like particles targeting EV-D68 has been developed. This vaccine has been proven effective in a mouse model in the prevention of AFM [7].

\section{Prognosis}

Only $5-39 \%$ of patients with AFM recover partially to completely (supplementary table 1). Most patients retain
Fig. 2 MRI of the spinal cord in a 3-year-old boy with GuillainBarré syndrome. a Sagittal contrast-enhanced $\mathrm{T} 1$ showing typical enhancing anterior caudal roots. $\mathbf{b}$ Subtraction of A with more clear depiction of enhancing caudal root. c Transverse $\mathrm{T} 1$ showing more clear enhancement of anterior motor roots

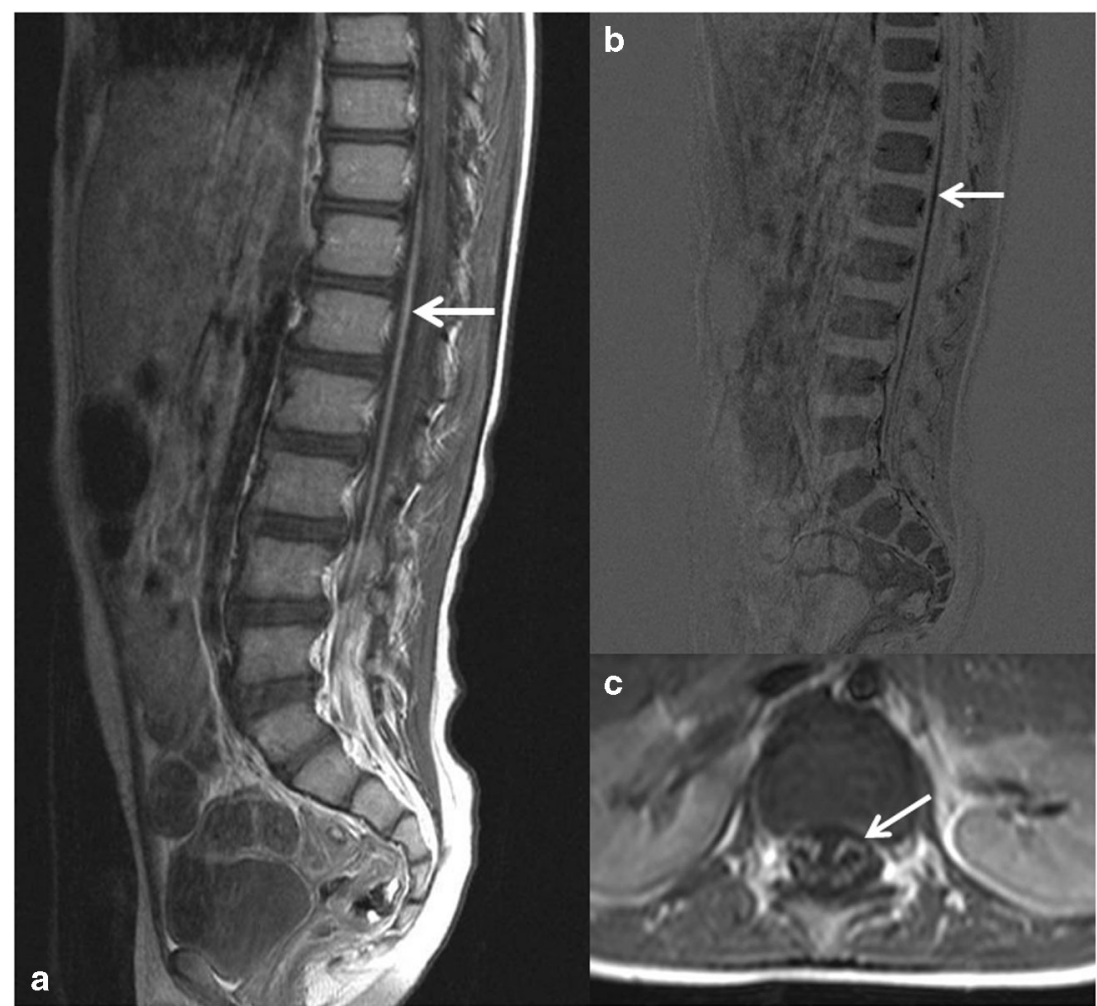


Fig. 3 MRI of the spinal cord in a 15-year-old boy with acute transverse myelitis, eventually diagnosed with relapsing remitting multiple sclerosis. a Sagittal T2 showing focal swelling of the spinal cord at level Th11-12. b Sagittal T1 showing contrast enhancement of the lesion

Fig. 4 MRI of a 13-year-old boy with a provisional diagnosis of acute demyelinating

encephalomyelitis. a Sagittal short tau inversion recovery (STIR) with edematous

cervicothoracic spinal cord from the level of C4. b Sagittal T1 of the spinal cord showing diffuse areas of slight enhancement. c Enhancement of mainly dorsal roots in a sagittal $\mathrm{T} 1$ of the lumbar spine. $\mathbf{d}$ and e Transverse $\mathrm{T} 2$ at the level of the pons (d) and thalamus (e) showing asymmetric hyperintense areas.
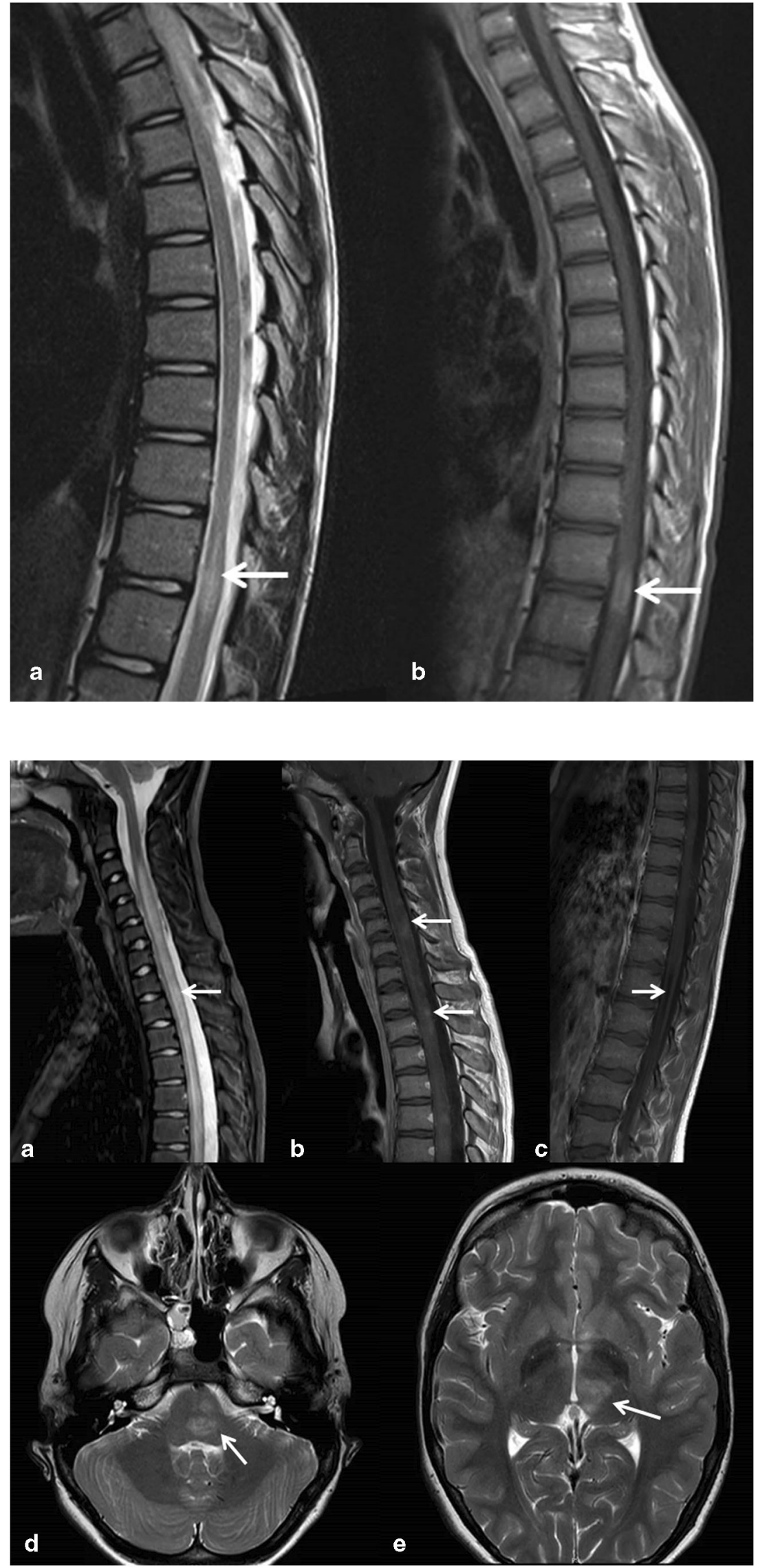
Table 3 Suggested workup for children with acute flaccid paralysis

$\begin{array}{ll}\text { Blood } & \text { Routine investigations (blood count, inflammatory parameters, creatine kinase, liver and renal function tests) } \\ & \text { Auto-antibodies (anti-MOG IgG, anti-AQP4, anti-GM1, anti-GQ1b) } \\ & \text { Oligoclonal bands (both serum and CSF) } \\ & \text { Microbiology: testing for enterovirus (including poliovirus), EBV, CMV, VZV, HEV, Zika virus* } \\ & \text { Routine investigations (cell count, protein, glucose) } \\ & \text { Oligoclonal bands (both CSF and serum) } \\ \text { CSF } & \text { Microbiology: testing for enterovirus, parechovirus, HSV, VZV, EBV } \\ & \text { Nasopharyngeal swab for enterovirus testing } \\ \text { Further microbiologic testing } & \text { Stool sample for enterovirus and } C \text {. jejuni testing } \\ & \text { Contrast-enhanced MRI of the brain and spine } \\ \text { Imaging } & \text { EMG with motor and sensory investigation of an affected limb } \\ \text { Neurophysiologic testing } & \end{array}$

$M O G$, myelin-oligodendrocyte glycoprotein; $A Q P 4$, aquaporin 4; GM1, ganglioside M1; GQ1b, ganglioside Q1b; $E B V$, Ebstain Barr virus; $C M V$, cytomegalovirus; $V Z V$, Varicella Zoster virus; $H E V$, hepatitis E virus; $C S F$, cerebrospinal fluid; $H S V$, Herpex Simplex virus; $E M G$, electromyography

*For patients that have traveled to or live in countries where Zika virus is prevalent

significant residual motor deficits, and prolonged need for ventilatory support is not uncommon. On follow-up, residual proximal weakness tends to be more severe than distal weakness, with severe atrophy occurring over time $[5,13,23,26$, 29, 48]. Cranial nerve deficits usually recover well over time. Death is uncommon but has been reported in immunocompromised patients, usually because of respiratory complications $[27,33]$. While not much is known about prognostic factors, more severe disability and weakness at nadir and the persistence of denervation seem to be associated with worse outcome. One study found a correlation between negative tests for EV-D68 at onset and better outcome, which made the authors speculate that viral clearance and host responses play a role in the severity of weakness in AFM [5]. Alternatively, these EV-D68-negative cases may be due to different etiologies associated with more favorable outcomes than cases confirmed to be associated with EV-D68.

\section{Conclusion and future perspectives}

AFM is a newly introduced term comprising AFP combined with longitudinally extensive lesions of the spinal cord on MRI. This syndrome resembles poliomyelitis and has been associated with different viruses, in particular EV-D68.

EV-D68 infection is usually asymptomatic or mildly symptomatic with respiratory illness, but it can be associated with anterior horn disease causing severe weakness, with only minimal improvement over time in most cases.

A major challenge lies in the propagation of correct diagnostic procedures, including viral testing on respiratory material in suspected AFM cases. Future research may identify risk factors for AFM in EV-D68-infected patients and will elucidate how these factors can be influenced.

We believe that worldwide collaboration between neurologists, radiologists, pediatricians, and microbiologists is necessary to make progress in preventing and treating this devastating childhood disease. Furthermore, we postulate that making AFM a notifiable disease in more countries can increase awareness among clinicians and governments.

Authors' Contributions J. Helfferich drafted and revised the manuscript. M. Knoester, C.C. van Leer-Buter, R.F. Neuteboom, L.C. Meiners and H.G.M. Niesters critically revised the manuscript. O.F. Brouwer critically revised the manuscript and supervised the writing of the manuscript. All authors gave final approval.

\section{Compliance with ethical standards}

Conflict of interest The authors declare that they have no conflict of interest.

Informed consent Additional informed consent was obtained from all individual participants for whom identifying information is included in this article.

Open Access This article is distributed under the terms of the Creative Commons Attribution 4.0 International License (http:// creativecommons.org/licenses/by/4.0/), which permits unrestricted use, distribution, and reproduction in any medium, provided you give appropriate credit to the original author(s) and the source, provide a link to the Creative Commons license, and indicate if changes were made.

\section{References}

1. Absoud M, Greenberg BM, Lim M et al (2016) Pediatric transverse myelitis. Neurology 87:S46-S52. https://doi.org/10.1212/WNL. 0000000000002820

2. Andersen EW, Kornberg AJ, Freeman JL et al (2017) Acute flaccid myelitis in childhood: a retrospective cohort study. Eur J Neurol 24: 1077-1083. https://doi.org/10.1111/ene.13345

3. Bonwitt J, Poel A, DeBolt C et al (2017) Acute flaccid myelitis among children - Washington, September-November 2016. MMWR Morb Mortal Wkly Rep 66:826-829. https://doi.org/10. 15585/mmwr.mm6631a2 
4. CDC (2017) Revision to the standardized surveillance and case definition for acute flaccid myelitis

5. Chong PF, Kira R, Mori H et al (2018) Clinical features of acute flaccid myelitis temporally associated with an enterovirus D68 outbreak: results of a nationwide survey of acute flaccid paralysis in Japan, August-December 2015. Clin Infect Dis 66:653-664. https://doi.org/10.1093/cid/cix860

6. Choudhary A, Sharma S, Sankhyan N et al (2010) Midbrain and spinal cord magnetic resonance imaging (MRI) changes in poliomyelitis. J Child Neurol 25:497-499. https://doi.org/10.1177/ 0883073809340918

7. Dai W, Zhang C, Zhang X et al (2018) A virus-like particle vaccine confers protection against enterovirus D68 lethal challenge in mice. Vaccine 36:653-659

8. Derrough T, Salekeen A (2016) Lessons learnt to keep Europe polio-free: a review of outbreaks in the European Union, European Economic Area, and candidate countries, 1973 to 2013. Euro Surveill 21:https://doi.org/10.2807/1560-7917.ES.2016.21. 16.30210

9. Dyda A, Stelzer-Braid S, Adam D, et al (2018) The association between acute flaccid myelitis (AFM) and Enterovirus D68 (EVD68) - what is the evidence for causation? Euro Surveill 23:https:// doi.org/10.2807/1560-7917.ES.2018.23.3.17-00310

10. Elrick MJ, Gordon-Lipkin E, Crawford TO et al (2019) Clinical subpopulations in a sample of North American children diagnosed with acute flaccid myelitis, 2012-2016. JAMA Pediatr 173:134 139. https://doi.org/10.1001/jamapediatrics.2018.4890

11. Force TUKAFPAT (2019) An increase in reports of acute flaccid paralysis (AFP) in the United Kingdom, 1 January 2018-21 January 2019: early findings. Euro Surveill 24:1900093. https:// doi.org/10.2807/1560-7917.ES.2019.24.6.1900093

12. Glass JD, Samuels O, Rich MM (2002) Poliomyelitis due to West Nile virus. N Engl J Med 347:1280-1281. https://doi.org/10.1056/ NEJM200210173471616

13. Gordon-Lipkin E, Munoz LS, Klein JL et al (2018) Comparative quantitative clinical, neuroimaging, and functional profiles in children with acute flaccid myelitis at acute and convalescent stages of disease. Dev Med Child Neurol 61:366-375. https://doi.org/10. 1111/dmen. 14030

14. Greninger AL, Naccache SN, Messacar K et al (2015) A novel outbreak enterovirus D68 strain associated with acute flaccid myelitis cases in the USA (2012-14): a retrospective cohort study. Lancet Infect Dis 15:671-682. https://doi.org/10.1016/S14733099(15)70093-9

15. Harvala H, Broberg E, Benschop K et al (2018) Recommendations for enterovirus diagnostics and characterisation within and beyond Europe. J Clin Virol 101:11-17. https://doi.org/10.1016/j.jcv.2018. 01.008

16. Helfferich J, Meiners LC, Brouwer OF (2017) Acute flaccid weakness associated with enterovirus D68. Eur J Paediatr Neurol 21: 594-595. https://doi.org/10.1016/j.ejpn.2017.02.001

17. Hixon AM, Yu G, Leser JS et al (2017) A mouse model of paralytic myelitis caused by enterovirus D68. PLoS Pathog 13:e1006199. https://doi.org/10.1371/journal.ppat.1006199

18. Hixon AM, Clarke P, Tyler KL (2017) Evaluating treatment efficacy in a mouse model of enterovirus D68-associated paralytic myelitis. J Infect Dis 216:1245-1253. https://doi.org/10.1093/infdis/ jix468

19. Hixon AM, Clarke P, Tyler KL (2019) Contemporary circulating enterovirus D68 strains infect and undergo retrograde axonal transport in spinal motor neurons independent of sialic acid. J Virol. https://doi.org/10.1128/JVI.00578-19

20. Hopkins SE, Elrick MJ, Messacar K (2018) Acute flaccid myelitiskeys to diagnosis, questions about treatment, and future directions. JAMA Pediatr Published: https://doi.org/10.1001/jamapediatrics. 2018.4896
21. Hovden IA, Pfeiffer HC (2015) Electrodiagnostic findings in acute flaccid myelitis related to enterovirus D68. Muscle Nerve 52:909910. https://doi.org/10.1002/mus.24738

22. Huang CC, Liu CC, Chang YC et al (1999) Neurologic complications in children with enterovirus 71 infection. N Engl J Med 341: 936-942. https://doi.org/10.1056/NEJM199909233411302

23. Hubner J, Kruse B, Christen HJ et al (2017) Acute flaccid myelitis in German children in 2016-the return of polio? Dtsch Arztebl Int 114:551-557. https://doi.org/10.3238/arztebl.2017.0551

24. Iverson SA, Ostdiek S, Prasai S et al (2017) Notes from the field: cluster of acute flaccid myelitis in five pediatric patients - Maricopa County, Arizona, 2016. MMWR Morb Mortal Wkly Rep 66:758760. https://doi.org/10.15585/mmwr.mm6628a4

25. Kane MS, Sonne C, Zhu S et al (2019) Incidence, risk factors and outcomes among children with acute flaccid myelitis: a populationbased cohort study in a California Health Network between 2011 and 2016. Pediatr Infect Dis J 38:667-672. https://doi.org/10.1097/ INF.0000000000002276

26. Kirolos A, Mark K, Shetty J et al (2018) Outcome of paediatric acute flaccid myelitis associated with enterovirus D68: a case series. Dev Med Child Neurol 61:376-380. https://doi.org/10.1111/dmen. 14096

27. Knoester M, Helfferich J, Poelman R et al (2019) Twenty-nine cases of enterovirus-D68-associated acute flaccid myelitis in Europe 2016: a case series and epidemiologic overview. Pediatr Infect Dis J 38:16-21. https://doi.org/10.1097/INF. 0000000000002188

28. Maloney JA, Mirsky DM, Messacar K et al (2014) MRI findings in children with acute flaccid paralysis and cranial nerve dysfunction occurring during the 2014 enterovirus D68 outbreak. Am J Neuroradiol 36:245-250. https://doi.org/10.3174/ajnr.A4188

29. Martin JA, Messacar K, Yang ML et al (2017) Outcomes of Colorado children with acute flaccid myelitis at 1 year. Neurology 89:129-137. https://doi.org/10.1212/WNL.0000000000004081

30. Marx A, Glass JD, Sutter RW (2000) Differential diagnosis of acute flaccid paralysis and its role in poliomyelitis surveillance. Epidemiol Rev 22:298-316

31. McKay SL, Lee AD, Lopez AS et al (2018) Increase in acute flaccid myelitis - United States, 2018. MMWR Morb Mortal Wkly Rep 67: 1273-1275. https://doi.org/10.15585/mmwr.mm6745e1

32. Messacar K, Schreiner TL, Maloney JA et al (2015) A cluster of acute flaccid paralysis and cranial nerve dysfunction temporally associated with an outbreak of enterovirus D68 in children in Colorado, USA. Lancet (London, England) 385:1662-1671. https://doi.org/10.1016/S0140-6736(14)62457-0

33. Messacar K, Schreiner TL, Van Haren K et al (2016) Acute flaccid myelitis: a clinical review of US cases 2012-2015. Ann Neurol 80: 326-338. https://doi.org/10.1002/ana.24730

34. Messacar K, Asturias EJ, Hixon AM et al (2018) Enterovirus D68 and acute flaccid myelitis-evaluating the evidence for causality. Lancet Infect Dis 18:e239-e247. https://doi.org/10.1016/S14733099(18)30094-X

35. Messacar K, Sillau S, Hopkins SE et al (2018) Safety, tolerability, and efficacy of fluoxetine as an antiviral for acute flaccid myelitis. Neurology 92:e2118-e2126. https://doi.org/10.1212/WNL. 0000000000006670

36. Morrey JD, Wang H, Hurst BL, et al (2018) Causation of acute flaccid paralysis by myelitis and myositis in enterovirus-D68 infected mice deficient in interferon alphabeta/gamma receptor deficient mice. Viruses 10:https://doi.org/10.3390/v10010033.

37. Okumura A, Mori H, Fee Chong P et al (2018) Serial MRI findings of acute flaccid myelitis during an outbreak of enterovirus D68 infection in Japan. Brain and Development 41:443-451. https:// doi.org/10.1016/j.braindev.2018.12.001

38. Poelman R, Scholvinck EH, Borger R et al (2015) The emergence of enterovirus D68 in a Dutch University Medical Center and the 
necessity for routinely screening for respiratory viruses. J Clin Virol 62:1-5. https://doi.org/10.1016/j.jcv.2014.11.011

39. Rao S, Messacar K, Torok MR et al (2016) Enterovirus D68 in critically ill children: a comparison with pandemic H1N1 influenza. Pediatr Crit Care Med 17:1023-1031. https://doi.org/10.1097/PCC. 0000000000000922

40. Ruggieri V, Paz MI, Peretti MG et al (2017) Enterovirus D68 infection in a cluster of children with acute flaccid myelitis, Buenos Aires, Argentina, 2016. Eur J Paediatr Neurol 21:884-890

41. Saltzman EB, Rancy SK, Sneag DB et al (2018) Nerve transfers for enterovirus D68-associated acute flaccid myelitis: a case series. Pediatr Neurol 88:25-30

42. Sarmast SN, Gowda VK, Ahmed M et al (2018) Acute flaccid myelitis-clustering of polio-like illness in the tertiary care centre in Southern India. J Trop Pediatr. https://doi.org/10.1093/tropej/ fmy052

43. Scheld WM, Marra CM, Whitley RJ (2004) Chapter 7: poliomyelitis, polio vaccines, and the postpoliomyelitis syndrome. In: J G (ed) 4th ed. Wolters Kluwer, Philadelphia, pp 112-124

44. Schieble JH, Fox VL, Lennette EH (1967) A probable new human picornavirus associated with respiratory diseases. Am J Epidemiol $85: 297-310$
45. Solomon T, Kneen R, Dung NM et al (1998) Poliomyelitis-like illness due to Japanese encephalitis virus. Lancet (London, England) 351:1094-1097

46. Wang SM, Lei HY, Huang MC et al (2006) Modulation of cytokine production by intravenous immunoglobulin in patients with enterovirus 71-associated brainstem encephalitis. J Clin Virol 37:47-52

47. Willison HJ, Jacobs BC, van Doorn PA (2016) Guillain-Barre syndrome. Lancet (London, England) 388:717-727. https://doi.org/10. 1016/S0140-6736(16)00339-1

48. Yea C, Bitnun A, Robinson J et al (2017) Longitudinal outcomes in the 2014 acute flaccid paralysis cluster in Canada. J Child Neurol 32:301-307. https://doi.org/10.1177/0883073816680770

49. Zhu F, Xu W, Xia J et al (2014) Efficacy, safety, and immunogenicity of an enterovirus 71 vaccine in China. N Engl J Med 370: 818-828. https://doi.org/10.1056/NEJMoa1304923

Publisher's note Springer Nature remains neutral with regard to jurisdictional claims in published maps and institutional affiliations. 